\title{
A efetividade da entrevista motivacional no autocuidado de pacientes com insuficiência cardíaca: revisão sistemática
}

\author{
The effectiveness of the motivational interview in self-care \\ of patients with heart failure: a systematic review
}

\author{
Thaís Medeiros Lima Guimarães' $\bullet$ Lyvia da Silva Figueiredo ${ }^{2} \bullet$ Nathália Sodré Velasco $^{3}$ \\ Rodrigo Leite Hipólito ${ }^{4}$ Gláucio Martins da Silva Bandeira ${ }^{5} \bullet$ Marina Einstoss Barbosa Siqueira ${ }^{6}$ \\ Ana Carla Dantas Cavalcanti ${ }^{7} \bullet$ Paula Vanessa Peclat Flores ${ }^{8}$
}

\begin{abstract}
RESUMO
Objetivo: Determinar a efetividade da intervenção entrevista motivacional no autocuidado dos pacientes com insuficiência cardíaca. Método:Trata-se de uma revisão sistemática, atualizada em julho de 2018, realizada nas bases MEDLINE, CINAHL, Cochrane Library, Joanna Briggs Institute Library, PubMed, LILACS e Scopus com os descritores: heart failure/insuficiência cardíaca, motivational interviewing/entrevista motivacional, self care/autocuidado e patient compliance/cooperação do paciente. Principais resultados: Foram identificados, inicialmente, 25 estudos em potencial para inclusão nesta revisão, no entanto, após serem aplicados os critérios de elegibilidade, 14 estudos foram encaminhados a dois revisores para avaliação metodológica, diante disso, três estudos foram selecionados após alcançarem rigor suficiente para inclusão e síntese. Conclusão: Percebe-se nos estudos, que é o impacto da comunicação na relação paciente x profissional de saúde, o que promove um elo fundamental e diretamente relacionado ao sucesso do tratamento. A síntese dos estudos evidenciou a efetividade da intervenção Entrevista Motivacional na adesão e no autocuidado dos pacientes com insuficiência cardíaca. Palavras-chave: Insuficiência Cardíaca; Entrevista Motivacional;Autocuidado e Cooperação do Paciente.
\end{abstract}

\begin{abstract}
Objective:To determine the effectiveness of the motivational interview intervention in the self-care of patients with heart failure. METHODS:This is a systematic review, updated in July 2018, based on the MEDLINE, CINAHL, Cochrane Library, Joanna Briggs Institute Library, PubMed, LILACS and Scopus databases, with the descriptors: heart failure/motivational interviewing/interview motivational, selfcare and patient compliance. MAIN RESULTS: We initially identified 25 potential studies for inclusion in this review; however, after eligibility criteria were applied, 14 studies were referred to two reviewers for methodological evaluation; three studies were selected after reaching enough accuracy for inclusion and synthesis. Conclusion: It is perceived in the studies, which is the impact of communication in the relation between patient and health professional, which promotes a fundamental link directly related to the success of the treatment. The synthesis of the studies evidenced the effectiveness of the Motivational Interview intervention in the adherence and self-care of patients with heart failure.
\end{abstract}

Keywords: Heart failure; Motivational Interview: Self-Care and Patient Compliance.

'E-mail:thaismedeiros.lima@hotmail.com. Enfermeira. Graduação pela Escola de Enfermagem Aurora deAfonso Costa/EEAAC da Universidade Federal Fluminense/UFF. 2E-mail: lyviafigueiredo@gmail.com. Enfermeira. Doutoranda em Ciências Cardiovasculares pela Universidade Federal Fluminense/UFF.

${ }^{3}$ E-mail: nathavelasco@hotmail.com. Enfermeira. Graduação pela Escola de Enfermagem Aurora de Afonso Costa/EEAAC da Universidade Federal Fluminense/UFF. ${ }^{4}$ E-mail: professorrlh@uol.com.br. Doutor em Enfermagem pela UERJ. Professor Adjunto II do Departamento de Enfermagem Médico-cirúrgica da Escola de Enfermagem Aurora de Afonso Costa/EEAAC da Universidade Federal Fluminense/UFF.

${ }^{5}$ E-mail: glauciobandeira@gmail.com. Pedagogo. Mestrando do Programa de Pós-Graduação em Ciências do Cuidado pela Escola de Enfermagem Aurora de Afonso Costa/EEAAC da Universidade Federal Fluminense/UFF.

${ }^{6}$ E-mail: ninaeinstoss@yahoo.com.br. Enfermeira. Graduação pela Escola de Enfermagem Aurora de Afonso Costa/EEAAC da Universidade Federal Fluminense/UFF. ${ }^{7}$ E-mail: anacarladc.uff@gmail.com Pós Doutora em Enfermagem pela UFRGS. Profa Associada do Departamento de Administração e Fundamentos de Enfermagem da Escola de Enfermagem Aurora de Afonso Costa/EEAAC da Universidade Federal Fluminense/UFF.

${ }^{8}$ E-mail: paulapeclat@gmail.com. Dra em Ciências Cardiovasculares pela UFF. Profa Adjunta II do Departamento de Enfermagem Médico-cirúrgica da Escola de Enfermagem Aurora de Afonso Costa da Universidade Federal Fluminense/UFF. 


\section{INTRODUÇÃO}

A insuficiência cardíaca (IC) é uma síndrome clínica complexa caracterizada por sintomas típicos que podem ser acompanhados de sinais resultantes de disfunção ou perda do músculo miocárdio, caracterizada por dilatação do ventrículo esquerdo ou hipertrofia ${ }^{(1)}$. Estimativas indicam que I5 milhões de pessoas são atingidas por IC na Europa ${ }^{(1-2)}$ e espera-se que nos próximos anos mais pessoas sejam acometidas devido aos avanços nos tratamentos de eventos cardíacos agudos e das altas taxas de envelhecimento da população(3).

Apesar de importante avanço em seu tratamento nas últimas décadas, a IC ainda é associada a readmissões hospitalares, baixa qualidade de vida, risco de mortalidade prematura e altos custos hospitalares ${ }^{(4)}$. Alguns fatores que contribuem para essas elevadas reinternações por descompensação da IC estão relacionados ao pouco conhecimento dos pacientes sobre a doença e tratamento, incapacidade para reconhecer sinais de piora, dificuldade de acesso aos serviços de saúde, idade avançada e ao suporte social inadequado ou isolamento social(5).

Nesse cenário preocupante, as estratégias de acompanhamento multidisciplinar na gestão do tratamento não farmacológico dos pacientes com IC e as orientações realizadas e reforçadas a cada consulta têm trazido benefícios no alcance e manutenção da estabilidade clínica e melhora do autocuidado e adesão ao tratamento(5).

$O$ enfermeiro e a equipe multiprofissional desempenham um papel importante na promoção da saúde e na educação dos comportamentos de autocuidado. Ao identificar os fatores que facilitam esses comportamentos, eles podem planejar intervenções para promover o autocuidado e assim melhorar a qualidade de vida nesses pacientes $^{(6)}$.

Compreender a adesão dos pacientes é um processo complexo que vai do cumprimento e acompanhamento do tratamento prescrito, incluindo o envolvimento e motivação dos pacientes na definição do seu plano de cuidados, em busca do bem-estar e saúde, representada por mudanças no estilo de vida que incluem o comparecimento às consultas e um maior controle das medicações. Ou seja, a melhora da adesão ao tratamento e do autocuidado é um processo que requer, principalmente, a mudança comportamental e a motivação do paciente ${ }^{(7)}$.

A necessidade de mudança comportamental e motivação dos pacientes inspirou a formulação de uma intervenção terapêutica chamada Entrevista Motivacional (EM) que tem como objetivo principal auxiliar o indivíduo nos processos de mudanças comportamentais, estimulando o comprometimento para a realização dessa mudança por meio de abordagem psicoterápica convincente e encorajadora ${ }^{(8)}$.

Como os pacientes com IC têm uma adesão ao auto- cuidado baixa e ineficaz, promover o autocuidado nesta população tornou-se uma das prioridades para os profissionais de saúde, o que levou a criação e implementação da estratégia Entrevista Motivacional( ${ }^{(9)}$.A mesma se trata de uma abordagem relativamente simples e com baixo custo, sendo baseada em princípios cognitivos como o entendimento dos problemas e as reações emocionais frente a eles, visando estabelecer alternativas para a modificação dos padrões de pensamentos implementando soluções e propondo intervenções terapêuticas individualizadas adequadas a cada estágio com vistas a aumentar a adesão ao tratamento( ${ }^{(8)}$.

Objetivou-se determinar o conhecimento produzido sobre o efeito e a efetividade da entrevista motivacional no autocuidado dos pacientes com insuficiência cardíaca.

\section{MÉTODO}

Trata-se de revisão sistemática realizada a partir das recomendações propostas no guia Preferred Reporting Items for Systematic Reviews and Meta- Analyses - PRIS$M A^{(10)}$. Uma busca inicial no PubMed, Cochrane e na biblioteca do The Joanna Briggs Institute (JBI) foi realizada para verificar a existência de alguma revisão sistemática sobre a temática, visando manter a originalidade do estudo. Também foi avaliado se lacunas de conhecimento específico refletem temática de interesse para $\circ$ universo científico e, por fim, concluiu-se que revisões sistemáticas que englobem a avaliação da efetividade da EM em pacientes com IC ainda não foram publicadas. Com isso, demonstrou-se evidente a necessidade em conduzir uma revisão sistemática sobre o tema.

Foi elaborado um protocolo baseado nas exigências internacionais sobre revisão sistemática e publicado na International Prospective Register of Systematic Reviews (PROSPERO) (https://www.crd.york.ac.uk/prospero), cuja número de registro é CRD42017080903.

A estratégia PICO é um modelo recomendado para simplificar a construção da pergunta do estudo e facilitar o processo de pesquisa. Neste modelo, a pergunta aplicada a uma situação prática deve ser estruturada em quatro elementos: Problema ou Paciente; Intervenção; Comparação ou Controle e Outcomes ou Desfecho( ${ }^{(1)}$. Neste estudo, considerou-se: P (Heart Failure/Insuficiência Cardíaca); I (Motivational Interviewing/Entrevista Motivacional); O (Patient Compliance/Cooperação do Paciente e Self Care/Autocuidado).

Foi considerada como pergunta do estudo: Qual o efeito da entrevista motivacional no autocuidado do paciente com insuficiência cardíaca em acompanhamento ambulatorial?

Os critérios de inclusão foram estudos em adultos maiores de 18 anos de idade, que abordem a temática da entrevista motivacional em pacientes com Insuficiência 
Cardíaca; com delineamento experimental ou quase experimental, como estudos sem randomização com grupo único pré e pós teste; séries temporais ou caso controle; indexados em bases de dados publicados em inglês, espanhol ou português, sem corte temporal, independente da área profissional de confecção do estudo. Excluíram-se estudos sem determinação de metodologia clara, teses e dissertações.

A estratégia de pesquisa visa encontrar estudos publicados e não publicados sem corte de tempo definido. A definição de descritores controlados foi referenciada nos termos Descritores em Ciências da Saúde (DeCS) e MESH (Medical Subject Headings). Os descritores controlados citados abaixo foram usados considerando os operadores booleanos "and" e "or" para pesquisa.

Devido as características específicas de cada base de dados, as estratégias de busca foram adaptadas de acordo com os objetivos e critérios de inclusão deste estudo. A busca dos artigos foi realizada no período de julho de 2018, conforme apresenta a estratégia PICO do quadro I:

A busca foi realizada nas seguintes bases de dados: MEDLINE, CINAHL, Cochrane Library, Joanna Briggs Institute Library, PubMed, LILACS e Scopus.

A revisão foi realizada em três estágios. No primeiro estágio foi elaborado o protocolo de estudo e realizada a busca nas bases de dados. Então, foram aplicados os critérios de elegibilidade nos resumos/abstracts/resumen. Em seguida, os dados dos artigos foram inseridos no sistema da JBI.

No segundo estágio, os textos completos foram avaliados quanto a validade metodológica, dois revisores cadastrados e treinados para o uso do sistema JBI Mastari. Os desacordos entre os revisores foram resolvidos através de discussão em grupo. Foram selecionados os estudos que atingiram avaliação positiva em pelo menos seis de dez itens do instrumento de avaliação crítica padronizado pelo JBI, que obtem informações detalhadas sobre o rigor metodológico dos estudos.

Os estudos selecionados pelos revisores foram também classificados de acordo com a avaliação de periódicos proposta pela CAPES, que passa por um processo de atualização. Esses veículos são enquadrados em extratos indicativos de qualidade - Al (o mais elevado); A2; BI; B2; B3; B4; B5; C (com peso zero). Além disso, os estudos foram classificados quanto ao nível de evidência. A prática baseada em evidências preconiza sistemas de classificação de evidências caracterizados de forma hierárquica, dependendo da abordagem metodológica adotada, que toma por base o delineamento do estudo. O nível I seria aplicado a evidências resultantes de metanálise de múltiplos ensaios clínicos controlados e randomizados; nível 2 para evidências obtidas em estudos individuais com delineamento experimental; nível 3 para evidências de estudos quase-experimentais; nível 4 para evidências de estudos descritivos (não experimentais) ou com abordagem qualitativa; nível 5 para evidências provenientes de relato de caso ou de experiência, e nível 6 para evidências baseadas na opinião de especialistas, em acordo categorização da Agency for Healthcare Research and Quality - $\mathrm{AHRQ}^{(12)}$. Neste estudo, também foi identificado o fator de impacto que é a principal métrica utilizada para avaliar as revistas científicas por todo o mundo ao contabilizar as citações recebidas; quanto maior o fator de impacto, melhor é considerada a revista e, consecutivamente, o padrão de qualidade dos artigos nela publicados.

No terceiro estágio, foi realizada a leitura e interpretação dos textos selecionados através do preenchimento de um instrumento previamente elaborado pelos autores para a obtenção das informações necessárias para análise da efetividade da entrevista motivacional utilizada nos estudos, tais como, objetivos, tipo de estudo, número de pacientes, desfecho e resultados encontrados.

Para reduzir os erros de interpretação dos resultados e do delineamento dos estudos analisados (viés), a busca foi realizada por três avaliadores, de forma independente, nas mesmas bases e com os mesmos descritores, apresentando ao fim, 100\% de concordância nos achados. Além disso, dois revisores verificaram a validação da qualidade metodológica de forma independente. Não houve discordância entre os mesmos.

QUADRO 1 - Estratégia PICO. Niterói, RJ, 2018

\begin{tabular}{|c|c|c|}
\hline \multicolumn{2}{|c|}{ MESH } & DECS \\
\hline $\begin{array}{c}\text { P } \\
(\mathbf{a n d})\end{array}$ & Heart Failure & Insuficiência Cardíaca \\
\hline $\mathbf{I}$ & Motivational Interviewing \\
$(\mathbf{a n d})$ & Entrevista Motivacional \\
\hline $\mathbf{C}$ & Patient Compliance & - OR \\
\hline $\mathbf{O}$ & Self Care & Adesão ao tratamento \\
& OR \\
\hline
\end{tabular}




\section{RESULTADOS}

\section{Seleção dos estudos}

A pesquisa identificou ao todo 25 estudos potencialmente relevantes e, após aplicação dos critérios de exclusão e eliminação dos estudos duplicados, I4 estudos foram selecionados e encaminhados aos avaliadores. Após esta avaliação, II estudos foram excluídos pelos avaliadores por não atingirem validação metodológica $(6 / 10)$, sendo três artigos incluídos nesta revisão sistemática, conforme indica a figura I. Destes três estudos, um era um estudo quanti-quali com nível de evidência 4, um ensaio clínico randomizado com nível de evidência 2 e um estudo de intervenção com nível de evidência 3.

\section{Características dos estudos}

As características dos estudos são relatadas no Quadro 2.

Dentre os estudos identificados, um ensaio clínico randomizado ${ }^{(14)}$ realizado em um único centro em 2016 nos EUA, teve como objetivo testar a eficácia de uma intervenção de entrevista motivacional em comparação aos cuidados habituais para melhorar o autocuidado, sintomas físicos e qualidade de vida de pacientes com IC. Os pacientes foram recrutados mediatamente após a alta, sendo que o grupo de intervenção recebeu uma visi- ta domiciliar e de 3 a 4 telefonemas de acompanhamento por uma enfermeira durante 90 dias, todos pautados na entrevista motivacional. Dos 67 participantes que completaram o estudo não houve diferenças entre os grupos na manutenção e confiança no autocuidado, sintomas físicos da IC ou qualidade de vida aos 90 dias. Em relação ao autocuidado, a diferença de médias nos grupos intervenção e controle, foram de $19,7 \pm 16,0$ e $12,| \pm| 8,3$ $(p=0,08)$, respectivamente na Manutenção do autocuidado e $26,6 \pm 20.8$ e $21,6 \pm 16,8 \quad(p=0,31)$, respectivamente para Confiança do autocuidado. Quando se calcula o tamanho do efeito, o d Cohen indica uma intervenção com efeito moderado ( $d$ de cohen $=0,44$ ).

Com o objetivo de determinar o efeito da educação baseada em entrevistas motivacionais no autocuidado de pacientes com insuficiência cardíaca com depressão, um estudo quase experimental ${ }^{(15)}$ realizado em 2017 no Irã, incluiu 82 pacientes com insuficiência cardíaca com depressão confirmada. Estes pacientes foram divididos em dois grupos, em que o grupo intervenção recebeu quatro sessões de educação comportamental de autocuidado com base nos princípios da entrevista motivacional e o grupo controle recebeu quatro sessões de educação convencional sobre o comportamento de autocuidado e, após 8 semanas, os pacientes de ambos os grupos foram

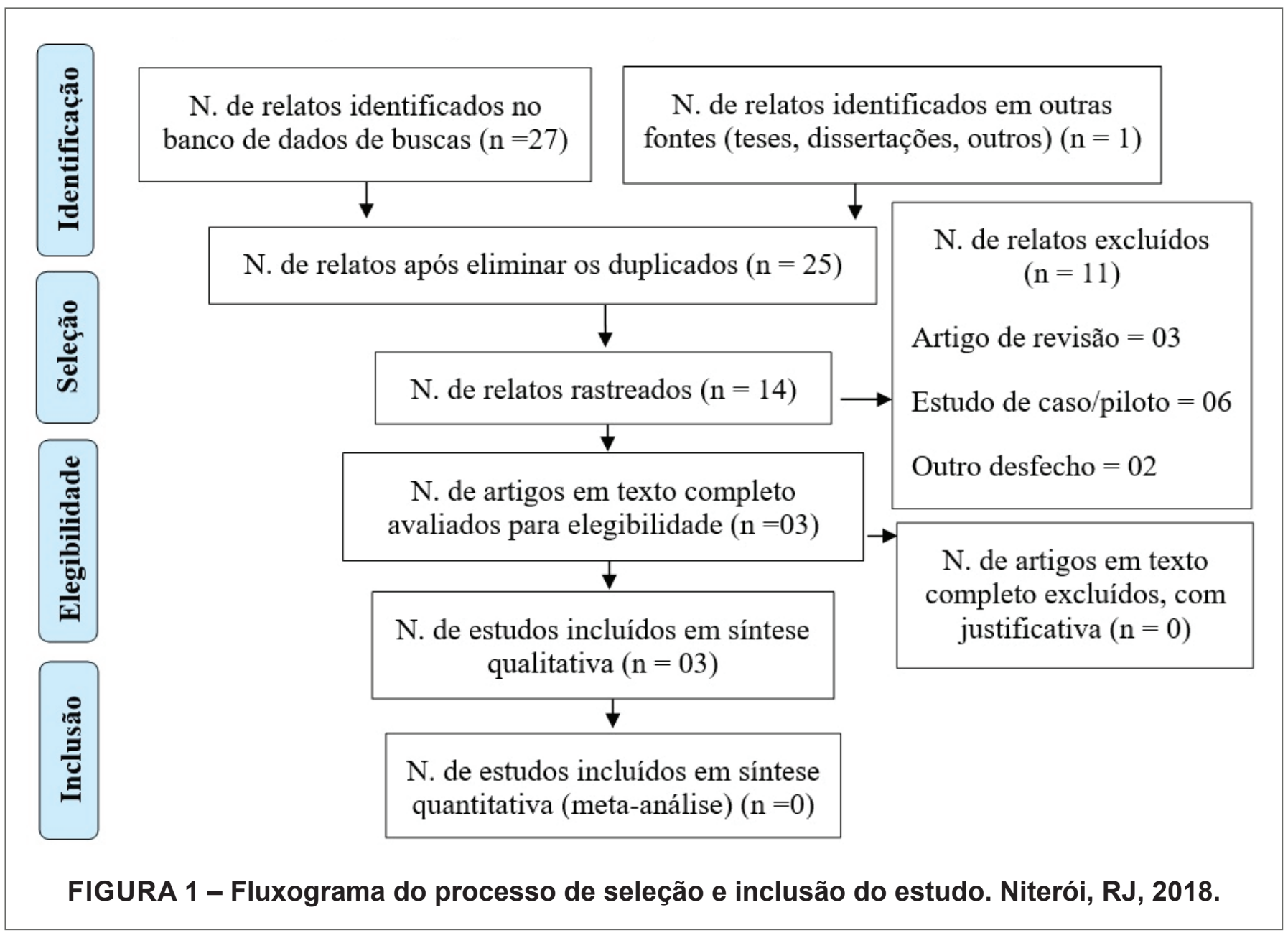


QUADRO 2 - Característica dos estudos. Niterói, RJ, Brasil, 2018.

\begin{tabular}{|c|c|c|c|}
\hline $\begin{array}{l}\text { Autor/País/ } \\
\text { Fator de } \\
\text { Impacto(FI)/ } \\
\text { Qualis Capes }\end{array}$ & $\begin{array}{l}\text { Desfechos/ } \\
\text { Tipo de } \\
\text { estudo(TE)/ } \\
\text { Nível de Evidência } \\
\text { (NE) }\end{array}$ & $\begin{array}{c}\text { Principais } \\
\text { características do } \\
\text { estudo/ } \\
\text { Amostra (N)/ } \\
\text { Tempo de seguimento } \\
\text { (T) }\end{array}$ & Resultados \\
\hline $\begin{array}{l}\text { Riegel et al. }{ }^{(13)} / \text { EUA } \\
\text { FI: } 2,429 \\
\text { Qualis } \\
\text { Enf: A2 } \\
\text { Med I: B1 }\end{array}$ & $\begin{array}{l}\text { Mecanismos de } \\
\text { eficácia da EM para } \\
\text { o autocuidado } \\
\text { TE: Quanti- } \\
\text { qualitativo. } \\
\text { NE: } 4\end{array}$ & $\begin{array}{l}\text { Identificar os } \\
\text { mecanismos de eficácia } \\
\text { da EM e sua relação } \\
\text { com o autocuidado; } \\
\text { N=41; T=90 dias }\end{array}$ & $\begin{array}{l}\text { Técnicas da EM evidenciadas: reflexão e reformulação; } \\
\text { empatia genuína, afirmação e humor; resolução de problemas } \\
\text { individualizados. Estas técnicas estimularam: definição de metas, } \\
\text { fala positiva, capacidade percebida para superar barreiras e } \\
\text { conversa sobre mudança. }\end{array}$ \\
\hline $\begin{array}{l}\left.\text { Masterson et al }{ }^{(14)}\right] \\
\text { EUA } \\
\text { FI: } 2,785 \\
\text { Qualis: Enf: A2; } \\
\text { Med I: B1 }\end{array}$ & $\begin{array}{l}\text { Melhora do } \\
\text { autocuidado } \\
\text { TE: Ensaio } \\
\text { controlado } \\
\text { randomizado } \\
\text { NE: } 2\end{array}$ & $\begin{array}{l}\text { Testar a eficácia da } \\
\text { EM no autocuidado } \\
\text { de pacientes com IC. } \\
\text { N=67, T=90 dias }\end{array}$ & $\begin{array}{l}\text { A intervenção foi pautada na resolução de resistência ou ambivalência } \\
\text { para mudar aspectos do autocuidado, através de conversas sobre } \\
\text { o planejamento de ações. Não houve diferenças entre os grupos na } \\
\text { manutenção do autocuidado, confiança no autocuidado, sintomas } \\
\text { físicos da IC ou qualidade de vida aos } 90 \text { dias. }\end{array}$ \\
\hline $\begin{array}{l}\text { Navidian et al(15)//rã } \\
\text { FI: } 2,785 \\
\text { Qualis: } \\
\text { Enf: A2; } \\
\text { Med 1: B1 }\end{array}$ & $\begin{array}{l}\text { Autocuidado e } \\
\text { depressão } \\
\text { TE: Quase } \\
\text { experimental } \\
\text { NE: } 3\end{array}$ & $\begin{array}{l}\text { Efeito da EM sobre } \\
\text { autocuidado em } \\
\text { pacientes com IC } \\
\text { depressivos. } \mathrm{N}=82 \text { dias }\end{array}$ & $\begin{array}{l}\text { A EM foi pautada na importância prática de equilíbrio decisório, } \\
\text { motivação intrínseca e apoio à auto eficácia. O aumento médio } \\
\text { nas pontuações gerais e as pontuações nas três subescalas do } \\
\text { autocuidado (manutenção, manejo e confiança) dos pacientes com } \\
\text { IC e depressão, foram maiores após a intervenção por EM }(p<0,05) \text {. }\end{array}$ \\
\hline
\end{tabular}

reavaliados para o mesmo desfecho. $\bigcirc$ estudo identificou que os escores totais e os escores manutenção, manejo e confiança do autocuidado nos pacientes com insuficiência cardíaca com depressão foram significativamente maiores após a educação baseada em entrevista motivacional do que após a autoavaliação convencional educação assistencial $(\mathrm{p}<0,05)$.

Como o desenvolvimento de estudos que utilizaram a entrevista motivacional apresentou resultados positivos, iniciou-se a indagação em relação aos mecanismos da EM que proporcionam a sua eficácia. Um estudo(13) americano realizado em 2017 apresentou como objetivo identificar os mecanismos que promovem a eficácia da EM frente às mudanças no autocuidado, através de um método misto.A intervenção por EM foi aplicada em 4I participantes, iniciando por uma visita domiciliar e continuou com 3 a 4 atendimentos telefônicos nos próximos 90 dias. Na visita domiciliar, foi utilizada a EM para ajudar os participantes a identificarem pelo menos dois objetivos que melhorariam o autocuidado em IC.A intervenção foi adaptada a esses objetivos e questões específicas basais do autocuidado em IC, identificadas através do índice de autocuidado em IC. $O$ estudo ocorreu através de um método misto, em que os dados quantitativos e os qualitativos foram triangulados para avaliar a congruência dos resultados no autocuidado, pré e pós intervenção. Parte das consultas foi gravada e os discursos dos pacientes foram utilizados na análise de dados. Todos os pacientes apresentaram melhoras nos índices de autocuidado (Ma-

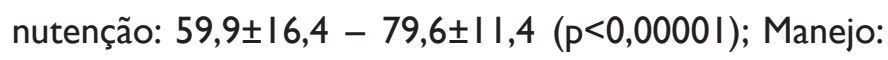
$50.9 \pm 28,1-68,4 \pm 23.3(p=0,002)$; Confiança: $54,65 \pm 22,2$ $-8 I, 23 \pm 17,9(p<0,000 I)^{(13)}$.

\section{DISCUSSÃO}

Este estudo identificou três artigos para pesquisa no qual foi possível perceber a congruência nos aspectos relacionados a necessidade de obtenção de novas informações acerca da EM.Todos os artigos se destacam pela busca de uma comprovação sobre a efetividade da EM associada a outros fatores.

Os estudos avaliados nos apontam fortes evidências do efeito da EM frente ao autocuidado do paciente com IC, demonstrando melhoras significativas nos escores de autocuidado no antes e depois das intervenções. No entanto, percebe-se nos estudos algo valioso, que é o impacto da comunicação na relação paciente $x$ profissional e saúde ${ }^{(13-15)}$, o que promove um elo fundamental e diretamente relacionado ao sucesso do tratamento ${ }^{(16)}$.

Os estudos destacam a importância da reflexão, conversa positiva sobre mudança, comunicação englobando empatia genuína, afirmação e humor promovendo capacidade para superar barreiras, resolução de problemas personalizada, estimulando um diálogo aberto para a definição de metas. Essas técnicas estimularam a abertura para a definição de metas, auto-fala positiva, capacidade percebida para superar barreiras e mudança conversa. Os mecanismos pelos quais as técnicas alcançaram os resultados desejados foram o desenvolvimento de dis- 
crepância e auto-eficácia, que são consistentes com os princípios da EM. Os autores concluiram que o presente estudo contribuiu para esclarecer o mecanismo pelo qual a EM facilita mudanças comportamentais, assim como, ressaltam que o uso da EM para discutir o autocuidado pode ajudar a superar as barreiras e envolver pacientes com IC na definição de metas para mudanças de comportamento ${ }^{(13)}$.

No processo de implementação do autocuidado, a decisão final da incorporação, ou não, de novos hábitos de vida, é inteiramente do paciente ${ }^{(17)}$. Quando nos deparamos com pacientes ambivalentes, a EM fornece subsídios que norteiam o profissional a auxiliar o paciente na tomada de decisão, evocando do cliente de forma equilibrada os prós e os contras, as vantagens e desvantagens do contexto ${ }^{(18-19)}$. A ambivalência é considerada não apenas como a relutância em fazer algo, mas a experiência de um conflito psicológico para decidir entre dois caminhos diferentes ${ }^{(18-20)}$.

Nesta vertente, todos os profissionais devem estar atentos a sua maneira de conduzir as consultas. Independente da utilização da EM o diálogo positivo, com empatia genuína e um pouco de bom humor podem tornar o ambiente mais agradável e susceptível a uma conversa sobre mudanças. Imposições ou listas de tarefas preconizadas pelos modelos tradicionais podem até surtir efeito, no entanto, precisamos estar atentos para técnicas de trabalho que apontem resultados duradouros e apoiem mudanças reais e efetivas.

A reflexão é a estratégia chave da $\mathrm{EM}^{(20)}$, e por este motivo a técnica é utilizada preconizando-se perguntas abertas na proporção de 3:I, em relação as perguntas fechadas. Perguntas abertas são aquelas que colocam o paciente na situação de precisar se expressar e não ficar refém dos corriqueiros "sim e não" nas consultas tradicionais ${ }^{(20)}$. Quando um paciente é convidado a refletir sobre seus comportamentos de saúde, é possível que ele mesmo se escute falando e com o apoio do entrevistador, reconheça onde pode redimensionar suas estratégias de autocuidado.

Os profissionais de saúde precisam estar atentos ao fato de que sua atuação ocorre durante a consulta e quando o paciente segue para sua casa, executará os cuidados da forma que ele decidir executar, por isso, uma consulta em que entrevistado e entrevistador dialogam e constroem juntos, tem grandes chances de se tornar mais efetiva no autocuidado. Promover estratégias que favoreçam a implementação do autocuidado tem impacto em outros desfechos, como a qualidade de vida(21).

A resolução de problemas de forma personalizada é a forma mais adequada de definir metas e ajustes no autocuidado de cada paciente. Estabelecimentos de padrões generalizados para doentes crônicos tornam a adesão às medidas de autocuidado ainda mais difíceis de serem alcançadas. Os pacientes com IC precisam incorporar diversos hábitos de vida, que somados, tornam-se muitas vezes difíceis e cansativos, no entanto, extremamente necessários. Neste contexto, conhecer a individualidade de cada um pode proporcionar o reconhecimento de adequações ou ajustes de rotinas que favoreçam, assim, a adesão ao autocuidado.

\section{CONCLUSÃO}

Este estudo avaliou a efetividade da intervenção Entrevista Motivacional na adesão e no autocuidado dos pacientes com insuficiência cardíaca através de uma revisão sistemática da literatura. Não foi possível realizar uma metanálise devido a diversidade dos métodos e desfechos, no entanto, este estudo foi capaz de demonstrar a forma no qual a Entrevista Motivacional pode atingir positivamente os pacientes com IC, proporcionando aos profissionais de saúde, fácil compreensão de novas estratégias que podem ser incorporadas nos atendimentos dos pacientes com IC. 


\section{REFERÊNCIAS}

I. Ponikowski P,Voors AA, Anker SD, Bueno H, Cleland JG, Coats AJ, et al. 2016 ESC Guidelines for the diagnosis and treatment of acute and chronic heart failure:The Task Force for the diagnosis and treatment of acute and chronic heart failure of the European Society of Cardiology (ESC). Developed with the special contribution. Eur J Heart Fail [Internet]. 2016 [acesso em 30 jul 2018]; 37(27): 89I-975. Disponível em: https:// academic.oup.com/eurheartj/article/37/27/2I 29/174892I.

2. Ponikowski P,Anker SD,AlHabib KF, Cowie MR, Force TL, Hu $\mathrm{S}$, et al. Heart failure: Preventing disease and death worldwide. ESC Hear Fail [Internet]. 2014 [acesso em 20 set 2018]; I: 4-25. Disponível em: https://onlinelibrary.wiley.com/doi/ epdf/10.1002/ehf2.12005

3. Sedlar N, Lainscak M, Martensson M, Strömberg A, Jaarsma T, Farkas J. Factors related to self-care behaviours in heart failure:A systematic review of European Heart Failure Self-Care Behaviour Scale studies. Europ J Card Nursing [Internet]. 2017 [acesso em 22 set 2018]; 16(4): 272-282. Disponível em: http:// journals.sagepub.com/doi/pdf/I0. I I77//4745 I 5 I I769 I644.

4. Rabelo ER, Aliti GB, Linch GFC, Sauer JM, Mello AMFS, Martins SM et al. Manejo não farmacológico de pacientes com insuficiência cardíaca descompensada: estudo multicêntrico - EMBRACE. Acta Paul Enf [Internet]. 2012 [acesso em 10 ago 2018]; 25(5):660-665. Disponível em: http://www.scielo.br/scielo.php?script=sci_arttext\&pi$\mathrm{d}=\mathrm{SO}$ I 03-2 I 002012000500003.

5. Mussi CM, Ruschel K, Souza EN, Lopes ANM, Trojahn MM, Paraboni CC, et al. Visita domiciliar melhora conhecimento, autocuidado e adesão na insuficiência cardíaca: ensaio clínico randomizado HELEN-II. Rev. Latino-Am. Enf [Internet]. 2013 [acesso em I5 ago 2018]; 21: [09 telas]. Disponível em: http://www.scielo.br/scielo.php?.pid=SO I 04- I I 6920 I 3000700004\&script=sci_arttext\&tlng=pt

6. Qavam SM, Sahebi A, Shohani M, Balavandi F, Qavam R, Tavan $\mathrm{H}$. Investigating the Effect of Education on Self-Care among Chronic Heart Failure Patients Admitted to Shahid Mostafa Khomeini Hospital of Ilam. Global J Health Science [Internet]. 2017 [acesso em I5 ago 2018]; 9 (5): 79-84. Disponível em: http:// www.ccsenet.org/journal/index.php/gjhs/article/view/60956.

7. Silva AF, Cavalcanti ACD, Malta M,Arruda CS, Gandin T, Fé A. et al.Adesão ao tratamento em pacientes com insuficiência cardíaca acompanhados por enfermeiras em duas clínicas especializadas. Rev. Latino-Am. Enf [Internet]. 2015 [acesso em 05 jul 2018]; 23(5): 888-94. Disponível em: http://www. revistas.usp.br/rlae/article/view/I06I55.

8. Bandinelli LP, Gonçalves HA, Fonseca RP.A entrevista motivacional e sua aplicabilidade em diferentes contextos: uma revisão sistemática. Rev Soc Psic RGS [Internet]. 2013 [acesso em 25 set 20I8]; I3(I): 26-34. Disponível em: http://www.sprgs.org. br/diaphora/ojs/index.php/diaphora/article/view/82

9. Rojas CMC, Rojas DNC, Reyes AMG. La entrevista motivacional como intervención de enfermería para promover el autocui- dado en pacientes con insuficiencia cardiaca en una institución de cuarto nivel en Bogotá, Colombia. Invest Enf: Imag y Desar [Internet]. 2013 [acesso em I 3 set 20I8]; I5(I): 3 I-49. Disponível em: http://revistas.javeriana.edu.co/index.php/imagenydesarrollo/article/view/6023.

10. Galvão TF, Pansani TSA, Harrad D. Principais itens para relatar Revisões sistemáticas e Meta-análises:A recomendação PRISMA. Epidemiol Serv Saúde [Internet]. 2015 [acesso em 13 set 2018]; 24 (2): 335-342. Disponível em: http://www. scielo.br/pdf/ress/v24n2/2237-9622-ress-24-02-00335.pdf.

I I. Ercole FF, Melo LS, Alcoforado CLGC. Revisão integrativa versus revisão sistemática. Rev Min Enferm [Internet]. 20।4[acesso em I4 ago 20I8]; I8(I):I-260. Disponível em: http://www.reme.org.br/artigo/detalhes/904.

12. Stetler CB, Morsi D, Rucki S, Broughton S, Corrigan B, Fitzgerald J, et al. Utilization-focused integrative reviews in a nursing service. Appl Nurs Res [Internet]. 1998 [acesso em 12 ago 2018]; II(4):195-206. Disponível em: https://doi. org/10.1016/S0897-1897(98)80329-7

13. Riegel B, Dickson VV, Garcia LE, Creber RM, Streur M. Mechanisms of change in self-care in adults with heart failure receiving a tailored, motivational interviewing intervention. Patient Ed Couns [Internet]. 2017 [acesso em 22 ago 20।8]; I00(2): 283-288. Disponível em: https://www.pec-journal.com/article/S0738-399I(16)30406-2/fulltext.

14. Creber RM, Patey M, Lee CS, Kuan A, Jurgens C, Riegel B. Motivational interviewing to improve self-care for patients with chronic heart failure: MITI-HF randomized controlled trial. Patient Ed Couns [Internet]. 2016 [acesso em 22 set 2018]; 99(2): 256-64. Disponível em: https://www.pec-journal. com/article/S0738-399I(I5)30067-7/fulltext.

I5. Navidian A, Mobaraki H, Shakiba M. The effect of education through motivational interviewing compared with conventional education on self-care behaviors in heart failure patients with depression. Patient Educ Couns [Internet]. 2017 [acesso em 10 set 2018]; I00(8): I 499- I 504. Disponível em: https://www. pec-journal.com/article/S0738-399 I(I7)30 I I4-3/fulltext.

16. Coriolano-Marinus MWL, Queiroga BAM, Ruiz-Moreno L, Lima LS. Comunicação nas práticas em saúde: revisão integrativa da literatura. Saúde Soc [Internet]. 2014 [acesso em 10 set 2018]; 23(4): I356-1369. Disponível em: http://www.scielo.br/ pdf/sausoc/v23n4/0104-1290-sausoc-23-4-1356.pdf.

17. Linden A, Butterworth SW.A Comprehensive Hospital-Based Intervention to Reduce Readmissions for Chronically III Patients:A Randomized Controlled Trial.The Am J Man Care [Internet]. 2014 [acesso em 10 set 2018];20(I0):783-92. Disponível em: https://www. ajmc.com/journals/issue/20 I/20 I4-vol20-n I0/a-comprehensive-hospital-based-intervention-to-reduce-readmissions-forchronically-ill-patients-a-randomized-controlled-trial.

18. Figlie NB, Guimarães LP. A Entrevista Motivacional: conversas sobre mudança. Acad Paul Psicol [Internet]. 2014 [acesso em 17 ago 2018]; 34(87): 472-89. Disponível em: http://pepsic.bvsalud.org/scielo.php?script=sci_arttext\&pi- 
$\mathrm{d}=\mathrm{S}|4| 5-7 \mid$ IX20 | $40002000 \mid$ | \&lng=pt\&nrm=iso\&tlng=pt

19. Brodie DA, Inoue A, Shaw DG. Motivational interviewing to change quality of life for people with chronic heart failure: A randomised controlled Trial. Int J Nurs Studies [Internet]. 2008 [acesso em 27 set 2018]; 45(4): 489-500. Disponível em: https://www.sciencedirect.com/science/article/pii/ S0020748906003 I78?via\%3Dihub

20. Rollnick S, Miller WR, Butler CC. Entrevista Motivacional no Cuidado da Saúde:Ajudando pacientes a mudar o comportamento. Porto Alegre:Artmed, 2009.

21. Carmo FR, Maruxo HB, Santos WA. Evidências científicas sobre a qualidade de vida dos pacientes com insuficiência cardíaca: revisão integrativa. Rev Enf Atual [Internet]. 2017 [acesso em 30 set 2018]; 82 (20): 87-94. Disponível em: https://revistaenfermagematual.com.br/uploads/revistas/20/I I.pdf 